\title{
Influence of the dissolution medium on the release of dehydroepiandrosterone from lipophilic suppositories
}

\author{
Regina KaspereK ${ }^{1 *}$, Krzysztof Galczynski², Magdalena NalesniaK ${ }^{1}$, \\ Karol IwaniaK ${ }^{1}$, Ewa PoleszaK ${ }^{1}$
}

\begin{abstract}
${ }^{1}$ Chair and Department of Applied Pharmacy, Medical University of Lublin, Chodzki 1, 20-093 Lublin, Poland
\end{abstract}
${ }^{2} 2^{\text {nd }}$ Chair and Department of Gynecology, Medical University of Lublin, Poland

\begin{tabular}{l}
\hline ARTICLE INFO \\
\hline Received 18 February 2014 \\
Accepted 04 March 2014 \\
\hline
\end{tabular}

\section{Keywords:}

dehydroepiandrosterone,

suppositories, release,

dissolution medium

\begin{abstract}
Suppositories with cocoa butter containing dehydroepiandrosterone (DHEA) without and with the addition of Span 80 and Tween 80 as surfactants with low and high HLB values were prepared. The physical properties and the drug content of all prepared suppositories were in accordance with the pharmacopoeial requirements. The release study tests in three dissolution media such as water, lactic acid solution at $\mathrm{pH} 4.2$ and phosphate buffer at $\mathrm{pH} 7.4$ were carried out. In acidic and alkalic media only about $10 \%$ and $27 \%$ of DHEA were released, respectively. The addition of Span 80 to the suppository mass did not improve the release process, but the addition of Tween 80 caused the increase in the amount of DHEA released in the acidic medium to about $35 \%$. The data showed that rectal administration of suppositories with DHEA based on cocoa butter caused about $30 \%$ availability and after vaginal administration, only topical activity can be expected. By the addition of Tween 80 to the suppository mass availability of DHEA of about $35 \%$ from vaginal suppositories can be achieved.
\end{abstract}

\section{INTRODUCTION}

Dehydroepiandrosterone (DHEA) is a sex steroid precursor secreted by the adrenals in humans and other primates. It converts intracellularly to bioactive androgens and estrogens [20]. DHEA and its sulfate metabolite (DHEAS) production decreases with advancing age [21]. DHEA and DHEAS play an important role in regulating body fat and the immune system. They also influence the circulation of lipoproteins and insulin sensitivity $[7,8,23]$. Therefore, they may protect against diseases of aging such as atherosclerosis, osteoporosis, decreased libidinal interest and diabetes $[5,6]$. There is much interest in the possible benefits of administering orally DHEA to postmenopausal women and for the purposes of maintaining sexual function, youthfulness, well-being and cognition [5,27]. The effect of intravaginal DHEA on the symptoms and signs of vaginal atrophy was also studied [27]. Symptoms such as vaginal dryness, itching, burning, irritation, and dyspareunia often occur as a result of a lack of estrogens $[9,22]$. Endometrial hyperplasia is the side effect observed in women receiving traditional estrogen replacement therapy that also increases the risk of carcinoma [3].

\footnotetext{
Corresponding author

* e-mail: regina.kasperek@umlub.pl
}

Vaginally administered DHEA may reduce symptoms of vaginal atrophy with improvements in sexual function in women who are estrogen deficient due to menopause [17-19, 26]. DHEA is an inactive precursor, which is transformed into active sex hormones (estrogens and/or androgens) only in specific cells and tissues, which possess the required enzymes [5]. Intravaginal DHEA, through local estrogen and androgen formation, causes a rapid effect without systemic exposure of other tissues, thus avoiding systemic effects of estrogens [18]. Labrie et al. [19] indicated that taking vaginal Prasterone suppositories (DHEA) daily causes the improvement in vaginal atrophy symptoms within 2 weeks.

In order to obtain both local and general actions of a drug, suppositories seem to be a convenient form of administration of the active substances [4, 19]. Cocoa butter is a frequently used lipophilic base for the preparation of lipophilic suppositories. It has a solid consistency at room temperature and it melts at body temperature. It is well tolerated and easy to obtain by various methods $[25,33]$. In order to examine the pharmaceutical availability of the drug in vitro studies, the amount of the drug substance released from the dosage form and dissolved in the surrounding liquid as well as the rate at which this process occurs should be determined [28]. The quantity of a drug released from suppositories depends on many factors such as drug solubility in the base, addition 
of surfactants and solubility of a drug in a dissolution medium $[1,12,30]$. The type and quantity of a surfactant included in the suppository base cause the increase or decrease of the drug release [29, 32]. The addition of surfactants in suppository formulations is used to improve availability of poorly soluble drugs. For a poorly soluble drug, solid dispersions and eutectic mixtures of a drug with inert carriers are used to enhance the rate of dissolution and absorption [2]. When the drug is of higher solubility in the suppository base then the quantity of the released substance is smaller because the drug has a tendency to be retained in the vehicle [10]. The nonionic surfactants in the suppository base incorporate some of the drug, which prevents its release [15]. There are many reports of studies on the effect of the addition of various types of surfactants on the drug release from the suppositories with lipophilic or hydrophilic bases $[12,14,15,30]$. The mechanism of action of surfactants is not completely known. It is quite common that they do not have a beneficial effect on the drug release [32].

The aim of this study was to evaluate the quantity of DHEA released from lipophilic suppositories prepared with and without the addition of a nonionic surfactant. DHEA is poorly soluble so the release studies were carried out in three media at different $\mathrm{pH}$ values.

\section{MATERIAL AND METHODS}

\section{Materials and Reagents}

Dehydroepiandrosterone was obtained from Fagron. Tween 80 and Span 80 were purchased from Fluka, Chemika, and cocoa butter and paraffin oil from Cefarm, Lublin, Poland. All the reagents and chemicals used were of analytical grade.

\section{Preparation of DHEA suppositories}

Suppositories weighing $2 \mathrm{~g}$ and containing $13 \mathrm{mg}$ of DHEA were prepared by hand rolling. The compositions of suppositories are presented in Table 1.

Table 1. Different suppositories compositions

\begin{tabular}{|l|c|c|c|}
\hline \multirow{2}{*}{ Name of Component } & \multicolumn{3}{|c|}{ Quantity (\%) per suppository of 2 g weight } \\
\cline { 2 - 4 } & S1 & S2 & S3 \\
\hline DHEA & 0.65 & 0.65 & 0.65 \\
\hline Cocoa oleum & 99.35 & 94.35 & 94.35 \\
\hline Tween 80 & - & 5 & - \\
\hline Span 80 & - & - & 5 \\
\hline
\end{tabular}

\section{Physical evaluation of DHEA suppositories \\ Weight uniformity test}

For each formulation, twenty suppositories were weighed and their average weight was calculated. Next, they were individually weighed using a weighing balance (Ohaus AV 513C, USA).

\section{Softening time determination}

The total deformation time was assayed by using the apparatus recommended by Polish Pharmacopoeia $9^{\text {th }}$ edition [28].

\section{Disintegration time}

The disintegration time was determined by using the disintegration tester ST 30 (Erweka, Germany) according to FP $9^{\text {th }}[28]$.

The data of physical parameters are given in Table 2 .

\section{Drug content analysis}

The drug content was analysed spectrophotometrically using the spectrophotometer UV-VIS (model Helios Omega UV-VIS, Spectro-Lab, Thermo scientific, England) with a 10 mm matched quartz cell.

\section{Calibration standards}

A stock solution of DHEA $(100 \mu \mathrm{g} / \mathrm{ml})$ was prepared by dissolving DHEA in the composed mixture (methanolwater- $0.1 \mathrm{~mol} / 1 \mathrm{NaOH}, 10: 5: 1, \mathrm{v} / \mathrm{v} / \mathrm{v}$ ). The calibration standards at a concentration of $5-50 \mu \mathrm{g} / \mathrm{ml}$ were obtained by the appropriate dilution of the stock solution with the composed mixture. The UV spectra of DHEA solution at a concentration of $10 \mu \mathrm{g} / \mathrm{ml}$ were measured spectrophotometrically. The maximum absorbance at a wavelength of $204 \mathrm{~nm}$ was found. The UV spectrum of DHEA at concentration of $32.5 \mu \mathrm{g} / \mathrm{ml}$ in mixture consisted of methanol, water and $\mathrm{NaOH}$ (10:5:1; $\mathrm{v}, \mathrm{v}, \mathrm{v})$ is shown in Figure 1. Suppositories containing a small amount just $13 \mathrm{mg}$ of DHEA have acceptable maximum absorbance at wavelength $204 \mathrm{~nm}$. The linear regression equation of the calibration curve equals $\mathrm{y}=0.0148 \mathrm{x}-$ 0.0004 . The coefficient of determination was $\mathrm{R}^{2}=0.9983$.

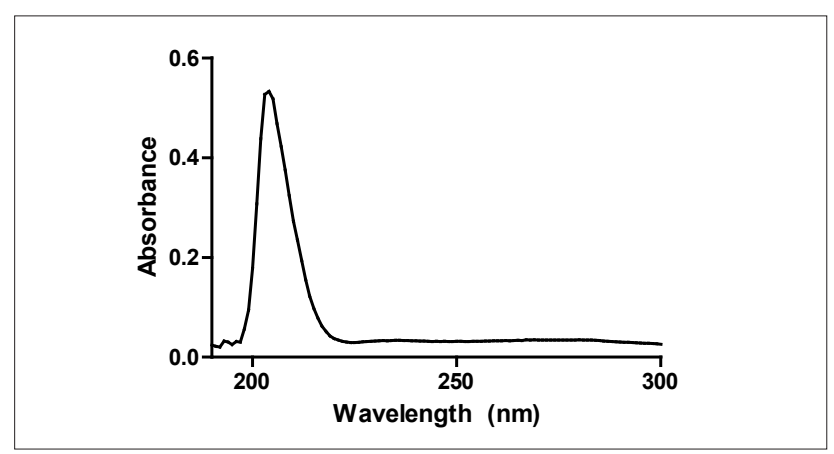

Figure 1. DHEA spectrum at concentration of $32.5 \mu \mathrm{g} / \mathrm{ml}$ in mixture consisting of methanol, water and $\mathrm{NaOH}(10: 5: 1 ; \mathrm{v}, \mathrm{v}, \mathrm{v})$

\section{Content uniformity}

Ten randomly selected suppositories were cut into small pieces. Accurately weighed $2 \mathrm{~g}$ sample pieces of suppositories were transferred into a $600 \mathrm{ml}$ beaker containing $160 \mathrm{ml}$ of the composed mixture. The content of the beaker was blended for one hour at $37^{\circ} \mathrm{C}$ into a water bath and filtered through $0.22 \mu \mathrm{m}$ membrane filter. Next, $3 \mathrm{ml}$ of obtained supernatant was diluted with the composed mixture to obtain $6 \mathrm{ml}$. The absorbance of the solution was measured spectrophotometrically at $204 \mathrm{~nm}$ against a blank prepared using a respective suppository without the drug. The mean content of the active substance $(n=6)$ was calculated from the equation obtained from the calibration curve. The data are given in Table 2. 
Table 2. Physical properties of suppositories prepared

\begin{tabular}{|l|c|}
\hline \multicolumn{1}{|c|}{ Test } & Results \\
\hline Weight (g) mean, SD & $1.99 \pm 3.17$ \\
\hline Disintegration time (min) & 16.16 \\
\hline Softening time (min), SD & $5.6 \pm 0.3$ \\
\hline Drug content (\%), SD & $103.57 \pm 11.04$ \\
\hline
\end{tabular}

\section{In vitro release test}

In the release studies water (W), lactic acid solution at pH 4.2 (LA), phosphate buffer at pH 7.4 (PB) or mixtures of these solutions with methanol $(1: 1, \mathrm{v} / \mathrm{v})$ were used as dissolution media.

\section{Calibration curve of DHEA}

The standard solutions of DHEA at concentrations $5-30 \mu \mathrm{g} / \mathrm{ml}$ in a mixture composed of a one of the dissolution medium (water, lactic acid at $\mathrm{pH} 4.2$ or phosphate buffer at $\mathrm{pH} 7.4)$ and methanol in ratio $2: 1(\mathrm{v} / \mathrm{v})$ were prepared. For each mixture the UV spectra of DHEA in concentration $10 \mu \mathrm{g} / \mathrm{ml}$ were determined and the maximum absorbance was found. Next, the calibration curves were prepared by measuring the absorbance of DHEA solutions spectrophotometrically at the respective wavelength. The values of the wavelengths, linear regression equations of the calibration curves and coefficients of determination are presented in Table 3.

Table 3. The values of wavelengths and parameters of the calibration curves for DHEA determination in different mixtures

\begin{tabular}{|l|c|c|c|}
\hline \multicolumn{1}{|c|}{$\begin{array}{c}\text { The type } \\
\text { of a mixture }\end{array}$} & $\begin{array}{c}\text { Water-methanol } \\
2: 1, \mathrm{v} / \mathrm{v}\end{array}$ & $\begin{array}{c}\text { Lactic acid } \\
\mathrm{pH} \text { 4.2-methanol } \\
2: 1, \mathrm{v} / \mathrm{v}\end{array}$ & $\begin{array}{c}\text { Phosphate buffer } \\
\mathrm{pH} \text { 7.4- } \text { - } \\
2: 1, \mathrm{v} / \mathrm{v}\end{array}$ \\
\hline Wavelength $(\mathrm{nm})$ & 194 & 195 & 199 \\
\hline $\begin{array}{l}\text { Linear regression } \\
\text { equation of the } \\
\text { calibration curve }\end{array}$ & $\begin{array}{c}\mathrm{y}=0.0326 \mathrm{x}+ \\
0,0325\end{array}$ & $\begin{array}{c}\mathrm{y}=0.0292 \mathrm{x}+ \\
0.0114\end{array}$ & $\mathrm{y}=0.0228 \mathrm{x}+0.0198$ \\
\hline $\begin{array}{l}\text { Coefficient of } \\
\text { determination }\left(\mathrm{R}^{2}\right)\end{array}$ & 0.9983 & 0.9976 & 0.9957 \\
\hline
\end{tabular}

In vitro release tests of DHEA from suppositories were carried out in the dissolution apparatus consisting of a baker and a stirrer [11] using $200 \mathrm{ml}$ of a dissolution medium at a temperature of $37 \pm 0.5^{\circ} \mathrm{C}$. The stirrer was rotated at the speed of $100 \mathrm{rpm}$.

A suppository was placed into the beaker containing a dissolution medium. The cloche stirrer was lowered to the height of 1-2 mm from the bottom of the beaker so that the suppository was under the stirrer. The samples, $5 \mathrm{ml}$ each, were withdrawn from the dissolution medium at time intervals after 5, 10, 20, 30, 40, 50 min and filtered through a $0.22 \mu \mathrm{m}$ millipore filter. The dissolution medium was replaced by $5 \mathrm{ml}$ of a fresh solution to maintain a constant volume. The filtered samples were diluted in water or lactic acid at $\mathrm{pH} 4.2$ or phosphate buffer at $\mathrm{pH} 7.4$ or methanol, respectively, depending on the dissolution medium in which the test was carried out. The samples were determined spectrophotometrically at the appropriate wavelength against a blank sample (suppository without drug). DHEA concentration was calculated based on calibration curves shown in Table 3.

\section{Statistical analysis}

Results are expressed as the mean of six experiments ( \pm SEM). For statistical evaluations, the data of release profiles of DHEA were assessed by the one way ANOVA analysis with post-hoc Turkey test, where $p<0.05$ was considered as statistically significant.

\section{RESULTS AND DISCUSSION}

The data in Table 2 shows that prepared suppositories fulfilled the pharmacopoeial requirements [20] of uniformity of mass for single dose preparations (the difference relative to the average weight was $\pm 5 \%$ ), disintegration time (up to $30 \mathrm{~min}$ ) and softening time (up to $15 \mathrm{~min}$ ), and the content of the active substance ranged from $85 \%$ to $115 \%$.

The results from suppositories without surfactants presented in Figure 2 show that within 50 min only 9.63\% or $12.05 \%$ of DHEA were released in LA at $\mathrm{pH} 4.2$ and water, respectively. The change of the dissolution medium to $\mathrm{PB}$ at $\mathrm{pH} 7.4$ caused the increase of the released substance up to $26.8 \%$, which confirmed better solubility of DHEA in an alkaline medium. These results indicated that bioavailability of DHEA from vaginal medium is poor and from a rectal medium it is only about $27 \%$. A small amount of the released substance is a result of its lipophilicity and low solubility in the used release fluids. The dissolution media used in the research imitate the environment in which vaginal or rectal suppositories are typically placed: LA solution at $\mathrm{pH} 4.2$ and $\mathrm{W}$ or $\mathrm{PB}$ at $\mathrm{pH} 7.4$, respectively $(16,24,28)$. The results obtained suggest that DHEA given in the vaginal form should have only a local effect, while pharmaceutical availability of the rectal form amounts to about $27 \%$.

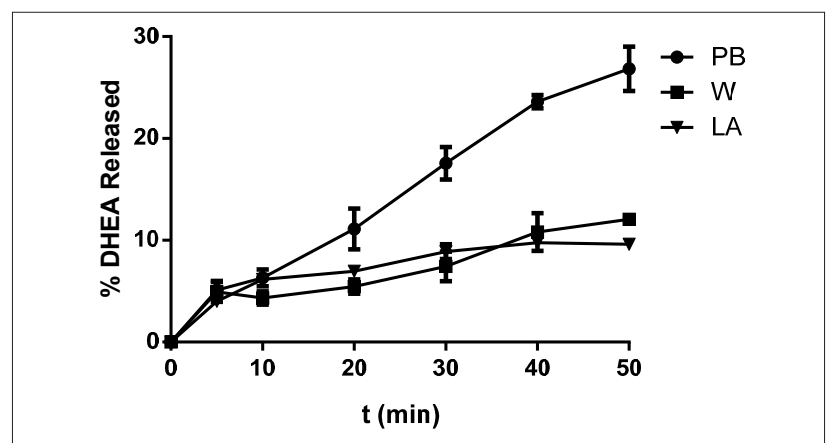

Figure 2. The amount of DHEA released from suppositories in three dissolution media $(n=6, p>0.05)$

An organic solvent or a surfactant can be added to a dissolution medium to increase the solubility of a drug. In the study performed by Sah and Saini [30] the addition of surfactant (Tween 80) into a dissolution medium at a low concentration $(1 \%)$ caused a greater release rate of indomethacin from suppositories, as compared to high concentration $(5 \%)$. The release rate of indomethacin was good from water-soluble suppositories bases in comparison with oil soluble bases.

In order to increase the solubility of the active substance and the possibility of comparing the dissolution profiles half of each dissolution medium was replaced by methanol. It turned out that the addition of an organic solvent to W, LA or PB caused the increase in the amount of the DHEA 
released to $67.57 \%, 76.65 \%$ and $95.3 \%$, respectively. The addition of methanol in the amount of $50 \%$ to all the dissolution media significantly affected the release of DHEA by increasing its solubility. The quantity of DHEA released increased significantly in each dissolution medium in the range from $56 \%$ to $68 \%$ (Fig. 3 ).

The additives in the suppository formulation can affect the increase or decrease of the release rate depending on the base [24]. In order to verify the effect of the surfactants to check the release process of DHEA were selected only two surfactants with different HLB values Span 80 (HLB $=4.3)$ and Tween $80(\mathrm{HLB}=15)$ in a high concentration of $5 \%$, typically added to the suppositories. In our study, the addition of Span 80 to the suppository mass did not improve the release process as the data in Figure 4 show. The quantity of DHEA released from suppositories with the addition of Span 80 decreased by about 19\% in PB and by 3\% in W, and in an acidic medium had no effect on the release process.

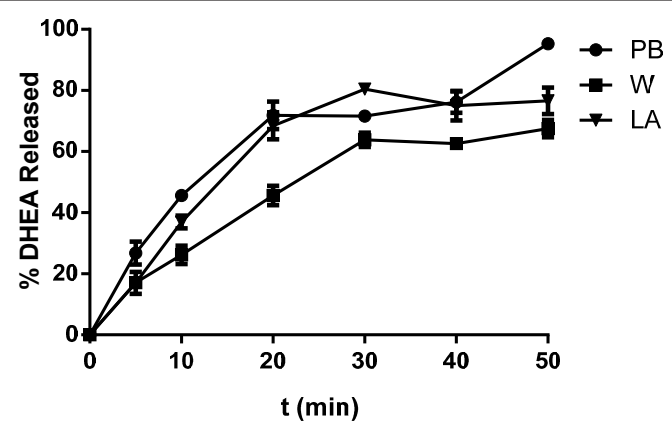

Figure 3. The amount of DHEA released from suppositories in three dissolution media with methanol $(n=6, p>0.05)$

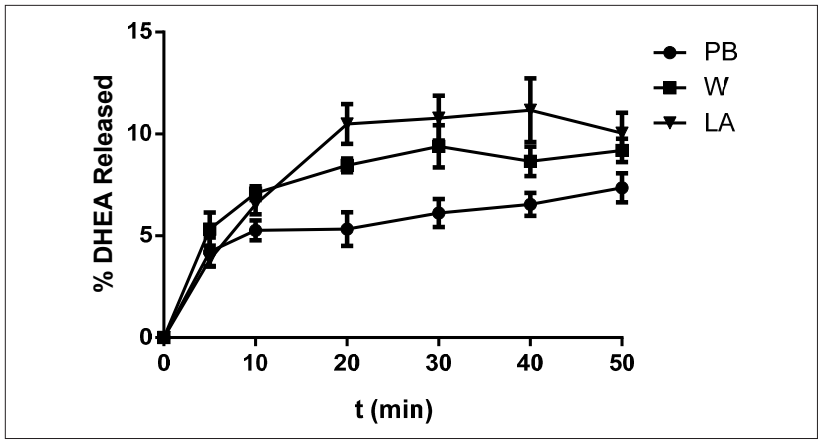

Figure 4. The amount of DHEA released from suppositories containing $5 \%$ of Span 80 in three dissolution media $(n=6, p>0.05)$

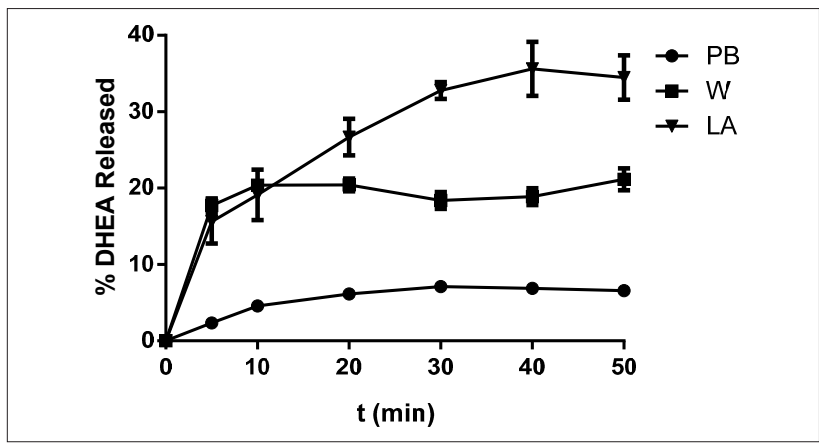

Figure 5. The amount of DHEA released from suppositories containing $5 \%$ of Tween 80 in three dissolution media $(n=6$, $\mathrm{p}>0.05)$
The addition of Tween 80 to the suppository mass caused an increase in the amount of the released substance depending on the $\mathrm{pH}$ of the dissolution medium (Fig.5). When the $\mathrm{pH}$ of the dissolution medium is decreased, the quantity of DHEA released increases and amounts to $34.46 \%$, $21.16 \%, 6.59 \%$ in LA, W and PB, respectively. From suppositories with Tween 80 the quantities of DHEA released in LA and $\mathrm{W}$ were $24 \%$ and $10 \%$ higher in LA and $\mathrm{W}$, respectively, comparing with suppositories without this surfactant. In alkaline medium (PB at $\mathrm{pH} 7.4$ ) the quantity of DHEA released amounts to only $6.59 \%$ from the suppository with the addition of Tween 80 . In this medium, there was a $20 \%$ decrease in the release.

The data obtained from the dissolution studies by Dal Zorro et al. [12] showed two glucose derived surfactants which caused a decrease in piroxicam release from lipophilic and hydrophilic suppositories. A more complex surfactant, a lauroyl macrogolglyceride, showed an increase in piroxicam availability from lipophilic suppositories at higher concentrations tested (15\% and $20 \%$ ).

Hargoli et al. [14] proved that among the formulations containing surfactants only Witepsol H15 with $0.5 \% \mathrm{w} / \mathrm{w}$ of Tween 80 and Witepsol W35 with $0.5 \%$ of cetylpyridinium chloride were suitable and caused nearly a complete release of naproxen from suppositories within 30 and 60 min, respectively.

In vitro release studies carried out by Ghorab et al. [13] showed a greater release of fenoterol bromochloride from hydrophilic bases than from fatty bases. Incorporating nonionic surfactants (Tween 60 and Span 20) into Witepsol H15 base caused an enhanced release rate of the drug at low surfactant concentrations.

In our study, as shown in Figures 6 and 7, in dissolution media with methanol, the release process from suppositories with an addition of surfactants runs worse in comparison with suppositories without surfactants. From suppositories containing Span 80 or Tween 80 in PB at pH 7.4 the quantity of DHEA released amounts to only $17.71 \%$ and $16.86 \%$, which indicates a decrease in the amount of the substance released by about $78 \%$. In water and LA at $\mathrm{pH} 4.2$ the quantity of the released DHEA decreased in the range from $18-25 \%$ and $20-25 \%$, respectively. Probably, the solubility of DHEA varies in acidic and alkaline media with the addition of a large amount of methanol.

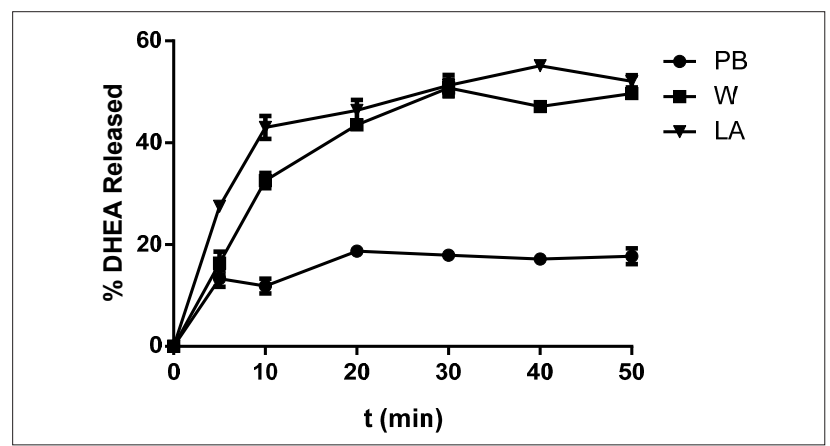

Figure 6. The amount of DHEA released from suppositories containing $5 \%$ of Span 80 in three dissolution media with methanol $(\mathrm{n}=6, \mathrm{p}>0.05)$ 


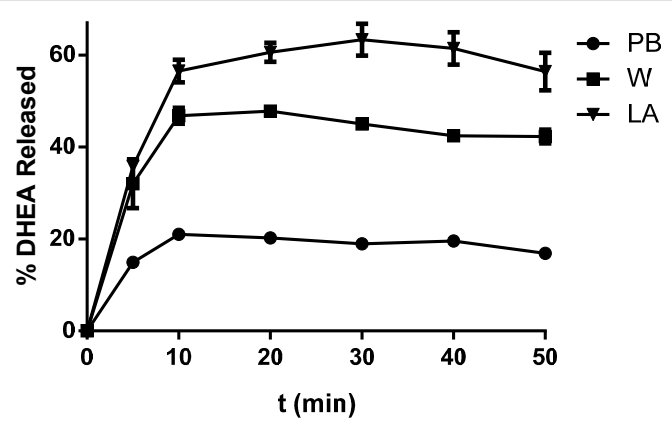

Figure 7. The amount of DHEA released from suppositories containing $5 \%$ of Tween 80 in three dissolution media with methanol $(n=6, p>0.05)$

\section{CONCLUSION}

The release of DHEA from the suppository with a cocoa butter base in acidic medium and water was poor and in the alkaline medium at $\mathrm{pH} 7.4$ it was slightly better. These studies showed that DHEA administered in vaginal suppositories could be used for local effect whereas after the administration of rectal suppositories about $30 \%$ bioavailability can be achieved. With the addition of Span 80 to the suppository mass, the amount of DHEA released from suppositories did not increase, but after the addition of Tween 80 about $35 \%$ bioavailability from vaginal suppositories was obtained.

\section{REFERENCES}

1. Azarmi S. et al.: Current perspectives in dissolution testing of conventional and novel dosage forms. Int. J. Pharm., 328(1), 12, 2007.

2. Babar A., Fares H. M., Plakogiannis F. M.: In vitro release of testosterone from suppository bases and in vivo absorption studies in human males. J. Pharm. Sci., 82(4), 389, 1993.

3. Beral V., Bull D., Reeves G.: Endometrial cancer and hormonereplacement therapy in the Million Women Study. Lancet, 365, 1543, 2005.

4. Bergogne-Bérézin E., Bryskier A.: The suppository form of antibiotic administration: pharmacokinetics and clinical application. J. Antimicrob. Chemother., 43(2), 177, 1999.

5. Buster J.E., Casson P.R. (2000). Biology and use in therapeutic intervention. in Menopause: Biology and Pathobiology. Lobo R. A., Kelsey J. and Marcus R. (editors). San Diego: Academic Press; p 628.

6. Casson P. R. et al.: Dehydroepiandrosterone (DHEA) replacement in postmenopausal women: Present status and future promise. Menopause, 4, 225, 1997.

7. Casson P. R. et al.: Oral dehydroepiandrosterone in physiologic doses modulates immune function in postmenopausal women. Am. J. Obstet. Gynecol., 169, 1536, 1993.

8. Casson P. R. et al.: Replacement of dehydroepiandrosterone enhances T-lymphocyte insulin binding in postmenopausal women. Fertil Steril, 63(5), 1027, 1995.
9. Castelo-Branco C. et al.: Management of post-menopausal vaginal atrophy and atrophic vaginitis. Maturitas, 52(Suppl. 1), 46, 2005.

10. Chicco D. et al.: Correlation of in vitro and in vivo paracetamol availability from layered excipient suppositories. Int. J. Pharm., 189, 147, 1999.

11. Czarnecki W., Kasperek R.: Patent RP Nr P337979 z 21.01.2000r. Biomed Wytwórnia Surowic i Szczepionek w Lublinie.

12. Dal Zorro M. et al.: Effect of the surfactant on the availability of piroxicam as a poorly hydrosoluble drug from suppositories. Pharmazie, 67(1), 37, 2012.

13. Ghorab D., Refai H., Tag R.: Preparation and evaluation of fenoterol hydrobromide suppositories. Drug Discov. Ther., 5(6), 311, 2011.

14. Hargoli S. et al.: Preparation and In vitro Evaluation of Naproxen Suppositories. Indian J. Pharm. Sci., 75(2), 143, 2013.

15. Kalmár É. et al.: Novel sample preparation method for surfactant containing suppositories: effect of micelle formation on drug recovery. J. Pharm. Biomed. Anal., 83, 149, 2013.

16. Karavana S. Y. et al.: Dissolution and histological studies on ordidazole vaginal formulation. Acta Pharma. Sci., 52, 430, 2010.

17. Labrie F. et al.: Effect of intravaginal dehydroepiandrosterone (Prasterone) on libido and sexual dysfunction in postmenopausal women. Menopause, 16(5), 923, 2009.

18. Labrie F. et al.: Intravaginal dehydroepiandrosterone (prasterone), a highly efficient treatment of dyspareunia. Climacteric, 14(2), 282, 2011.

19. Labrie F. et al.: Intravaginal dehydroepiandrosterone (Prasterone), a physiological and highly efficient treatment of vaginal atrophy. Menopause, 16(5), 907, 2009.

20. Labrie F. et al.: Is Dehydroepiandrosterone a hormone? J. Endocrinol., 187,169, 2005.

21. Labrie F. et al.: Marked decline in serum concentrations of adrenal C19 sex steroid precursors and conjugated androgen metabolites during aging. J. Clin. Endocrinol. Metab., 82, 2396, 1997.

22. Lynch C.: Vaginal estrogen therapy for the treatment of atrophic vaginitis. J. Womens Health (Larchmt), 18(10), 1595, 2009.

23. Nestler J. E. et al.: Dehydroepiandrosterone reduces serum low density lipoprotein levels and body fat but does not alter insulin sensitivity in normal men. J. Clin. Endocrinol. Metab., 66, 57, 1988.

24. Niraj et al.: Effect of Adjuvants on the Release Pattern of Suppositories Containing Paracetamol. RJCES, 1(1), 19, 2013.

25. Odeniyi M. A., Jaiyeoba K. T.: Effects of interacting variables on the release properties of chloroquine and aminophylline suppositories. Trop. J. Pharm. Res., 3(1), 285, 2004.

26. Panjari M., Davis S.R.: DHEA for postmenopausal women: a review of the evidence. Maturitas, 66(2), 172, 2010.

27. Panjari M., Davis S.R.: Vaginal DHEA to treat menopause related atrophy: a review of the evidence. Maturitas, 70(1), 22, 2011.

28. Polish Pharmacopoeia IXth ed., vol. I, PTF Warsaw (2011).

29. Realdon N. et al.: Effects of surfactant characteristics on drug availability from suppositories. Pharmazie, 63(6), 459, 2008.

30. Sah M. L., Saini T.R.: Formulation development and release studies of indomethacin suppositories. Indian J. Pharm. Sci., 70(4), 498, 2008.

31. Varshney H. M., Chatterjee A.: Formulation, evaluation and in-vitro release characteristics of zaltoprofen suppositories. Asian J. Pharm. Clin. Res., 5(4), 235, 2012.

32. Varshney H. M., Tanwar Y. S.: Effect of different surfactants on the release pattern of cocoa butter suppositories containing flurbiprofen sodium. Acta Pharm. Sci., 52, 129, 2010.

33. Verma P. et al.: Routes of drug administration. Int. J. Pharm. Studies Res., 1(1), 54, 2010. 